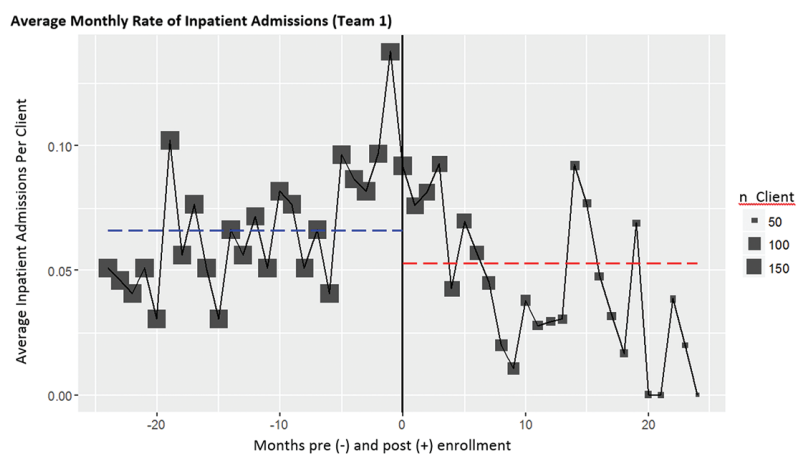

Abstract 1033 Figure 3 Average monthly rate of inpatient admissions (team 1)

physician continuity) included both descriptive and statistical modelling approaches from a pre/post comparison perspective. Results Across all teams there were strong improvements in self-reported experience for both patients and providers. Some teams demonstrated reduced acute care utilisation and cost, and higher continuity with a family physician. Better outcomes were linked with teams delivering on more elements of the Managing Complex Change model: having a vision, skills, incentives, adequate resources and an action plan.

Conclusions The evaluation demonstrates that the teams have improved care for their patients. Lessons learned from this evaluation will be critical for the Initiative moving forward, and also others working with similar populations. Recommendations from the evaluation for implementing system-level improvement initiatives will be discussed, as well as recommendation for implementing measurement systems with complex patients.

\section{THE EMERGENCY LAPAROTOMY COLLABORATIVE: SCALING UP AN IMPROVEMENT BUNDLE FOR HIGH RISK SURGICAL PATIENTS}

${ }^{1}$ Carol Peden, ${ }^{2}$ Geeta Aggarwal, ${ }^{2}$ Nial Quiney, ${ }^{3}$ Anne Pullyblank, ${ }^{4}$ Tim Stephens. ${ }^{1}$ Keck Medicine of USC, US; ${ }^{2}$ Royal Surrey Hospital NHS Trust, UK; ${ }^{3}$ North Bristol NHS Trust, UK; ${ }^{4}$ Queen Mary, University of London, UK

\subsection{6/bmjoq-2017-|HI.27}

Background Emergency intra-abdominal laparotomy is a common surgical procedure. Mortality is high with $11 \%-15 \%$ of patients dying within 30 days of surgery. Complication rates are also high and $>25 \%$ of patients remain in hospital for >20 days. A previous study, ELPQuIC, successfully used a care bundle to reduce mortality in four hospitals.

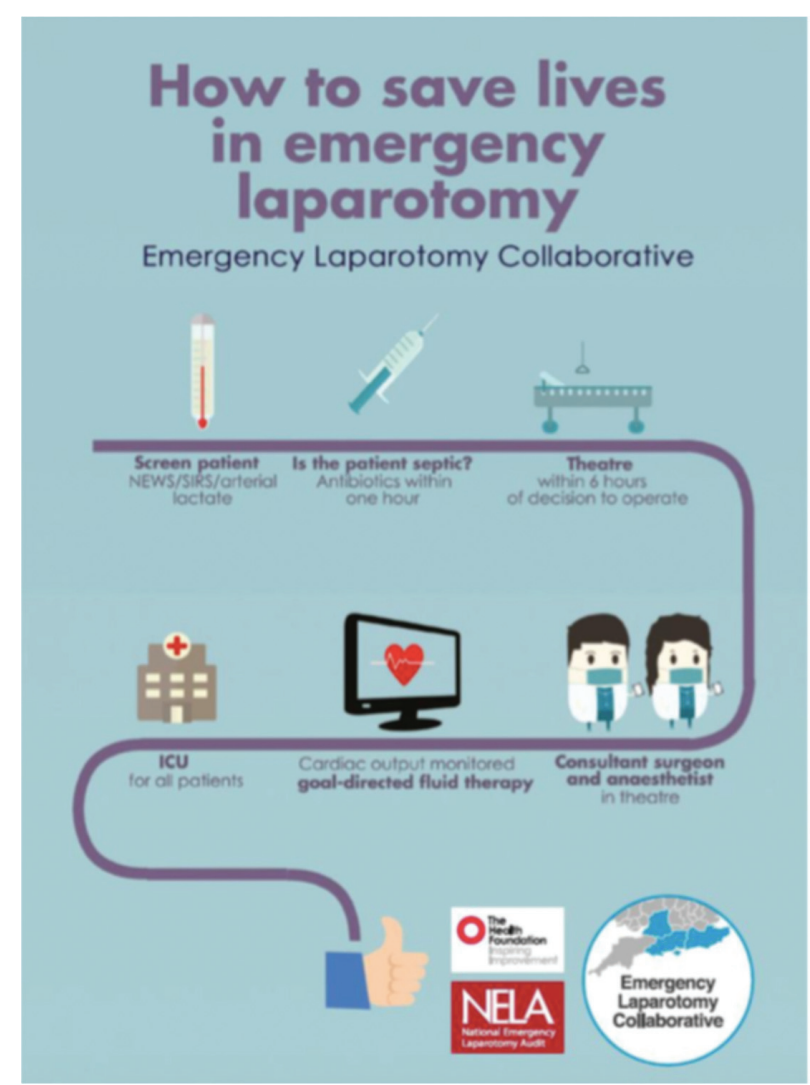

Abstract 1040 Figure 1 Diagram of the 'ELPQulC' Care Bundle

Objectives The aim of the Emergency Laparotomy Collaborative was to scale implementation of the ELPQuIC bundle to 24 NHS Trusts within three Academic Networks to reduce mortality, complications and length of stay.

Methods We used the IHI Breakthrough Series Collaborative Model to bring $100+$ staff together over two years with 5 large events and 4 local quality improvement events. Data collection was through the National Emergency Laparotomy Audit (NELA). Economic analysis was undertaken. Using NELA data we distributed comparative dashboards showing care bundle adherence and patient outcomes quarterly. The collaborative model enabled Trusts to share progress through dialogue, group reflection and celebration of success.

Results 5793 patients had an emergency laparotomy between October 2015 and December 2016. Crude mortality decreased from $9.8 \%$ to $8.7 \%$ and length of stay decreased by 1.3 days. There were significant improvements in delivery of care bundle components. Economic analysis showed potential savings of $£ 2 \mathrm{M}$ in 15 months, primarily through decreased length of

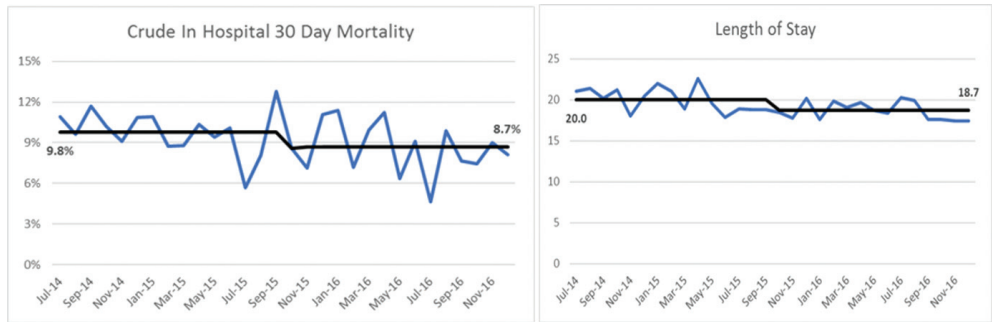

Abstract 1040 Figure 2 Run charts of mortality and length of stay from all hospitals. Intervention began October 2015. 


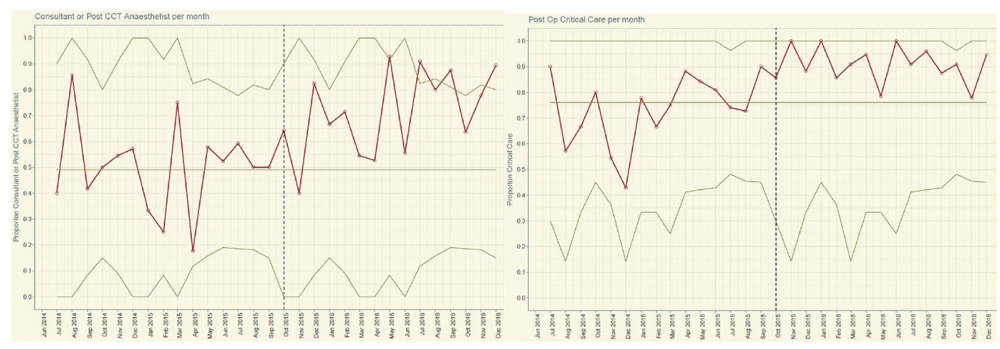

Abstract 1040 Figure 3 SPC charts from one hospital showing improvement in senior anaesthetist presence in theater and increase in number of patients going to critical care after surgery. Dotted line marks start of intervention.

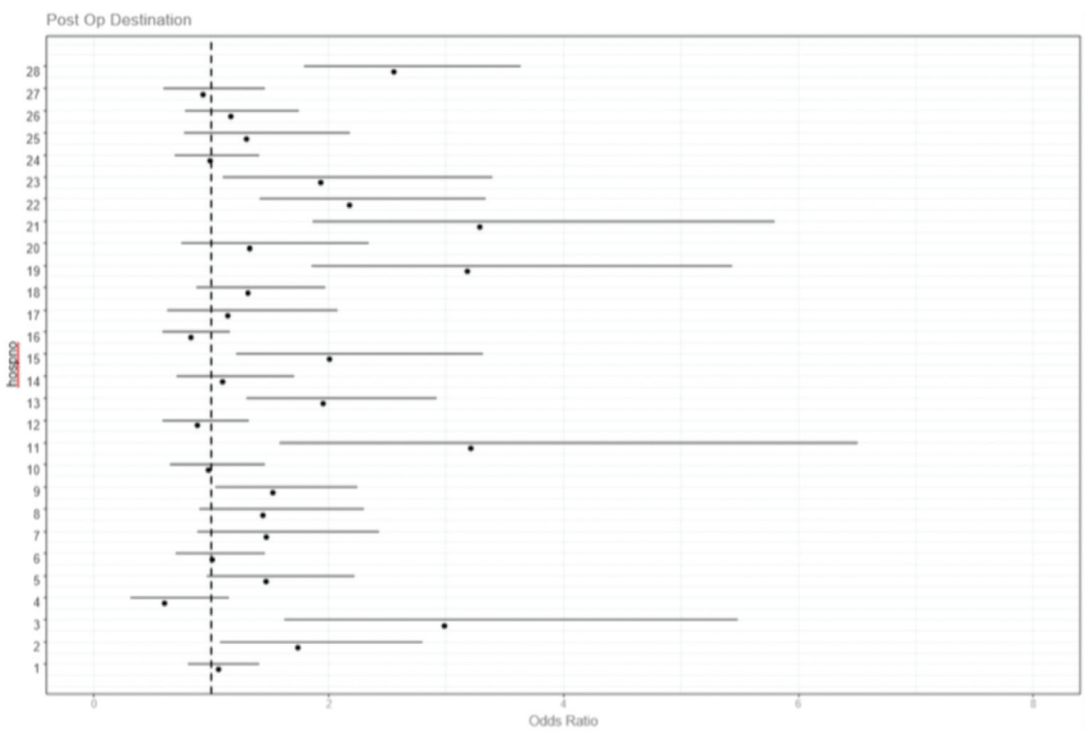

Abstract 1040 Figure 4 Odds ratios showing the increased likelihood of a patient receiving the implementation or outcome. Where the horizontal line is no longer in touch with the vertical line, a significant change has taken place. This charts shows how the change in post-op critical care has been replicated across the majority of hospitals. The chart includes analysis of historic data and the first 15 months of prospective collected data.

stay. The collaborative promoted innovation with ideas such as 'virtual peer review' emerging.

Conclusions Implementation of the ELPQuIC care bundle improved process delivery resulting in better outcomes for emergency laparotomy patients across 25 NHS Trusts. QI promotion through a BTS model fostered collaboration and innovation.

\section{REFERENCES}

1. National Emergency Laparotomy Audit. The Second Patient Report 2016. Available from NELA 2016. http://www.nela.org.uk/reports.

2. Al-Temimi, Griffee M, Ennis TM et al. When is death inevitable after emergency laparotomy? Analysis of the American College of Surgeons National Surgical Quality Improvement Program database. J Am Coll Surg 2012;215:503-11.

3. Saunders D, Murray D, Varley S, Pichel A, Peden CJ. Variations in mortality after emergency laparotomy: the first report of the UK Emergency Laparotomy Network. BJA 2012;109:368-375.

4. Huddart S, Peden CJ, Swart M et al. Use of a pathway quality improvement care bundle to reduce mortality after emergency laparotomy. BJS 2015;102:57-66.

\section{DECREASING UNNECESSARY BLOOD BANK TESTING FOR LAPAROSCOPIC HYSTERECTOMY}

Anne Que, Aalok Agarwala. Massachusetts General Hospital, Department of Anaesthesia, Critical Care and Pain Medicine, US

10.1136/bmjoq-2017-|HI.28
Background Reducing unnecessary preparation of blood components decreases cost and improves value. Standard surgical blood order schedules (SSBOS), which make recommendations on which procedures require a type and screen ( $\mathrm{T}$ and $\mathrm{S}$ ), have been shown to help reduce variability and improve patient safety. We explore how evidence-based interventions preoperatively optimise the process.

Objectives Our aim was to standardise the process and implement an evidence-based process improvement to reduce unnecessary $\mathrm{T}$ and $\mathrm{S}$ samples for laparoscopic hysterectomy cases.

Methods After IRB approval, we reviewed data from January 2014 to February 2016. Outcomes followed were: 1) T and S samples and 2) blood transfusion rates. Interventions were: 1) termination of routine ordering of $\mathrm{T}$ and $\mathrm{S}$ samples by preanaesthesia evaluation team (4/2015); and 2) implementation of a SSBOS guideline within a new EHR system (4/2016). We used statistical process control and descriptive statistics for analysis.

Results At baseline, of 615 laparoscopic hysterectomy procedures, $\mathrm{T}$ and $\mathrm{S}$ obtained for 490 procedures (78\%), 21 patients $(3 \%)$ received at least 1 unit of blood. Of 490 procedures after Intervention-1, $\mathrm{T}$ and $\mathrm{S}$ obtained for 300 cases (61\%), 18 patients (3\%) received transfusion. Of 552 procedures after Intervention-2, $\mathrm{T}$ and $\mathrm{S}$ obtained for 144 cases 